\title{
Surfing gravitational waves: can bigravity survive growing tensor modes?
}

\author{
Luca Amendola ${ }^{1}$, Frank Koennig ${ }^{1}$, Matteo Martinelli ${ }^{1}$, Valeria Pettorino ${ }^{1}$, Miguel Zumalacarregui ${ }^{1}$ \\ ${ }^{1}$ Institut für Theoretische Physik, Ruprecht-Karls-Universität Heidelberg, Philosophenweg 16, 69120 Heidelberg, Germany
}

The theory of bigravity offers one of the simplest possibilities to describe a massive graviton while having self-accelerating cosmological solutions without a cosmological constant. However, it has been shown recently that bigravity is affected by early-time fast growing modes on the tensor sector. Here we argue that we can only trust the linear analysis up to when perturbations are in the linear regime and use a cut-off to stop the growing of the metric perturbations. This analysis, although more consistent, still leads to growing tensor modes that are unacceptably large for the theory to be compatible with measurements of the cosmic microwave background (CMB), both in temperature and polarization spectra. In order to suppress the growing modes and make the model compatible with CMB spectra, we find it necessary to either fine-tune the initial conditions, modify the theory or set the cut-off for the tensor perturbations of the second metric much lower than unity. Initial conditions such that the growing mode is sufficiently suppresed can be achieved in scenarios in which inflation ends at the $\mathrm{GeV}$ scale.

\section{INTRODUCTION}

Evidence from an increasing number of cosmological observables favours an accelerating universe at late times [1 9 . This era of accelerated expansion may be due to novel gravitational physics, which will be tested by ongoing and future experiments [10]. This possibility has triggered vigorous interest in alternative theories of gravity [8, 11, 12, Any modification of gravity requires new degrees of freedom (dof). Since the theory of a massless graviton is unique, new dofs are often gained by adding new fields. The simplest possibility is the addition of a scalar field, typically resulting in theories belonging to the Horndeski class [13, 14] or beyond [15, 16].

Formulating a theory of massive gravity has been a long standing problem in theoretical physics due to the difficulties to incorporate the right degrees of freedom. The linear Fierz-Pauli theory had been developed long time ago [17, but until recently all non-linear completions introduced the so called Bouleware-Deser (BD) ghost [18, an extra dof that makes the theory not viable. Despite the difficulties, a class of healthy theories has been recently identified [19] in which a specific choice of the potential terms makes the theory ghost-free [20]. All these theories of massive gravity describe an interaction of two tensor fields in which the second one, the so called reference metric, is fixed. While massive gravity only allows static solutions on homogeneous backgrounds [21], a bimetric theory with a dynamical reference metric does not introduce the BD ghost and describes dynamical cosmologies [22 24] (see also the reviews [25, 26]). Cosmological solutions in these bimetric theories often allow for self-acceleration without the introduction of a cosmological constant [27] and were successfully compared to observations at background level [27-29].

Many bigravity theories are however affected by gradient instabilities in their scalar sector, as has been shown by studies of the linear perturbations [30 32] (see Refs. [31, 33] for derivations of the equations Refs. [34 38, for discussion of their dynamics). Stable evolution can be achieved only in a two parameters class of models known as Infinite-Branch Bigravity (IBB) [35]. In IBB, the reference metric (in keeping with common usage, we keep referring to the second metric as reference metric even if in reality is dynamical; we also use the notation $f$-metric) is contracting during the radiation and most of the matter era, until it undergoes a bounce at low redshift and begins to expand, coinciding with the onset of accelerated expansion in the physical metric without the need for a cosmological constant. The early time contraction of the reference metric makes tensor perturbations grow with time in IBB theories, as it was first shown in Ref. [36, 39] (see also [31, 40] for modified tensor perturbation equations). This growing mode couples to the physical metric and severely modifies its dynamics, leading to observable consequences.

In this paper we will investigate the effects of these large tensor perturbations on the Cosmic Microwave Background (CMB) and possible mechanisms to make the theory compatible with current observations. The perturbations in the reference metric grow very fast and rapidly become non-linear. At this point we will assume that tensor perturbations stabilize, modeling this effect by introducing a cut-off in the perturbations of the reference metric. Despite this treatment, the tensor growing mode significantly affects the evolution of the physical metric, and the consequences can be seen as an enhancement of both temperature and polarization spectra on low multipoles. These effects cannot be sufficiently reduced by varying the bigravity or other cosmological parameters: making the theory viable requires either fine tuning of the initial conditions, lowering the cut-off or modifying the theory. As it will be shown below, sufficient suppression of the growing mode can be achieved by an inflationary mechanism that produces Hubble-scale tensor perturbations at an energy scale of order few $\mathrm{GeV}$. 


\section{BIGRAVITY}

We start with the action of the form 22

$$
\begin{aligned}
S & =-\frac{M_{g}^{2}}{2} \int d^{4} x \sqrt{-g} R(g)-\frac{M_{f}^{2}}{2} \int d^{4} x \sqrt{-f} R(f) \\
& +m^{2} M_{g}^{2} \int d^{4} x \sqrt{-g} \sum_{n=0}^{4} \beta_{n} e_{n}(X)+\int d^{4} x \sqrt{-g} \mathcal{L}_{m}
\end{aligned}
$$

where $e_{n}(X)$ are the elementary symmetric polynomials of the eigenvalues of the matrices $X_{\gamma}^{\alpha} \equiv \sqrt{g^{\alpha \beta} f_{\beta \gamma}}, M_{g}$ and $M_{f}$ are the Planck masses for $g_{\mu \nu}$ and $f_{\mu \nu}$, respectively, $m$ is the mass scale of the graviton, $\beta_{n}$ are arbitrary constants and $\mathcal{L}_{m}=\mathcal{L}_{m}(g, \psi)$ is the matter Lagrangian. Throughout the paper we will use a mostly plus metric signature convention and natural units in which the speed of light $c$ is set to one.

Here $g_{\mu \nu}$ is the standard metric coupled to matter fields in the $\mathcal{L}_{m}$ Lagrangian, while $f_{\mu \nu}$ is an additional dynamical tensor field. In the following we express masses in units of the Planck mass $M_{g}$ and the mass parameter $m^{2}$ will be absorbed into the parameters $\beta_{n}$. Varying the action with respect to $g_{\mu \nu}$, one obtains the following equations of motion:

$$
G_{\mu \nu}+\frac{1}{2} \sum_{n=0}^{3}(-1)^{n} m^{2} \beta_{n}\left[g_{\mu \lambda} Y_{(n) \nu}^{\lambda}(X)+g_{\nu \lambda} Y_{(n) \mu}^{\lambda}(X)\right]=T_{\mu \nu}
$$

where $G_{\mu \nu}$ is Einstein's tensor, and the expressions $Y_{(n) \nu}^{\lambda}(X)$ are defined as

$$
\begin{aligned}
Y_{(0)} & =I \\
Y_{(1)} & =X-I[X] \\
Y_{(2)} & =X^{2}-X[X]+\frac{1}{2} I\left([X]^{2}-\left[X^{2}\right]\right) \\
Y_{(3)} & \left.=X^{3}-X^{2}[X]+\frac{1}{2} X\left([X]^{2}-\left[X^{2}\right]\right)\right) \\
& -\frac{1}{6} I\left([X]^{3}-3[X]\left[X^{2}\right]+2\left[X^{3}\right]\right)
\end{aligned}
$$

where $I$ is the identity matrix and $[. .$.$] is the trace operator.$

Varying the action with respect to $f_{\mu \nu}$ we get

$$
\bar{G}_{\mu \nu}+\sum_{n=0}^{3} \frac{(-1)^{n} m^{2} \beta_{4-n}}{2 M_{f}^{2}}\left[f_{\mu \lambda} Y_{(n) \nu}^{\lambda}\left(X^{-1}\right)+f_{\nu \lambda} Y_{(n) \mu}^{\lambda}\left(X^{-1}\right)\right]=0
$$

where the overbar indicates $f_{\mu \nu}$ curvatures. Notice that $\beta_{0}$ acts as a pure cosmological constant, which is however not required to satisfy current observations. Finally, the rescaling $f \rightarrow M_{f}^{-2} f, \beta_{n} \rightarrow M_{f}^{n} \beta_{n}$ allows us to assume $M_{f}=1$ in the following (see [4]). Additionally, from now on we absorb the graviton mass $m$ into the constants $\beta_{i}$.

We assume now a cosmological spatially flat FRW metric:

$$
d s^{2}=a^{2}(\tau)\left(-d \tau^{2}+d x_{i} d x^{i}\right)
$$

where $\tau$ represents the conformal time and a dot will represent the derivative with respect to it. The second metric is chosen also in a spatially FRW form

$$
d s_{f}^{2}=-\left[\dot{b}(\tau)^{2} / \mathcal{H}^{2}(t)\right] d \tau^{2}+b(\tau)^{2} d x_{i} d x^{i}
$$

where $\mathcal{H} \equiv \dot{a} / a$ is the conformal Hubble function and $b(\tau)$ is the 'scale' factor associated with the second metric $f$. This form of the metric $f_{\mu \nu}$ ensures that the equations satisfy the Bianchi constraints (see e.g. [24]).

The background equations for the two metrics have been obtained and discussed at length in several papers [27, 28, 35, 42. Here we summarize the main properties in the notation of [35]. Defining $r(\tau) \equiv b(\tau) / a(\tau)$ as the ratio of 
the two scale factors, the background equations can be conveniently written as a first order system of two equations for $r(t)$ and $\mathcal{H}$ :

$$
\begin{aligned}
2 \mathcal{H}^{\prime} \mathcal{H}+\mathcal{H}^{2} & =a^{2}\left(B_{0}+B_{2} r^{\prime}-w_{t o t} \rho_{t o t}\right), \\
r^{\prime} & =\frac{3 r B_{1} \Omega_{t o t}\left(1+w_{t o t}\right)}{\beta_{1}-3 \beta_{3} r^{2}-2 \beta_{4} r^{3}+3 B_{2} r^{2}},
\end{aligned}
$$

where the prime denotes derivative with respect to $N \equiv \log a[28,29], w_{t o t}$ denotes the equation of state (EOS) corresponding to the total density parameter $\Omega_{\text {tot }}$ and the functions $B_{0}(\tau), B_{1}(\tau), B_{2}(\tau)$ are related to the $\beta_{i}$ and $r(\tau)$ as follows:

$$
\begin{aligned}
& B_{0}(\tau)=\beta_{0}+3 \beta_{1} r+3 \beta_{2} r^{2}+\beta_{3} r^{3} \\
& B_{1}(\tau)=\beta_{1}+3 \beta_{2} r+3 \beta_{3} r^{2}+\beta_{4} r^{3} \\
& B_{2}(\tau)=\beta_{1}+2 \beta_{2} r+\beta_{3} r^{2} .
\end{aligned}
$$

For simplicity the time dependence of $B_{0,1,2}$ will be understood from now on. The Friedmann equation (i.e. the $(0,0)$ component of Eq. (2)) gives

$$
3 \mathcal{H}^{2}=a^{2}\left(\rho_{\text {tot }}+B_{0}\right)
$$

and by combining with the $(0,0)$ component of Eq. 7) we obtain a useful relation between $\mathcal{H}$ and the ratio $r(\tau)$ :

$$
\mathcal{H}^{2}=\frac{a^{2} B_{1}}{3 r} .
$$

Finally, the combination of the last two expressions for $\mathcal{H}$ provide $\Omega_{t o t}(\tau)=1-\frac{B_{0}}{B_{1}} r(\tau)$ which can be inserted in Eq. (11) to produce a closed differential equation for $r(\tau)$ alone.

The behavior of the background solutions depends on the choice of the $\beta_{i}$ constants and on the initial value of $r$. We denote solutions with the same $\beta_{i}$ but different initial conditions as branches of the same theory. In Ref. [29] it was shown that for each choice of $\beta_{i}$ only two branches exist that agree with a standard cosmological early time evolution (like a matter dominated era at early times) and allow for physical solutions (e.g. $\rho, \mathcal{H}>0)$. In the first branch, $r$ evolves from $r=0$ to a de Sitter point at a finite value $r_{c}>0$. These branches, however, suffer from scalar instabilities 30]. Only choosing $\beta_{2}=\beta_{3}=0$ and the second type of branches in which $r$ evolves from $r \rightarrow \infty$ in the asymptotic past towards a de Sitter point at a constant $r_{c}>0$, allows for stable scalar perturbations and is compatible with background data [35]: we dubbed this case infinite-branch bigravity (IBB). Note that even though an additional non-vanishing effective cosmological constant $\beta_{0}$ is viable, we assume $\beta_{0}=0$ since it would not affect the early-time evolution and is not required in order to fit observational data (see [28, 29]). From now on we restrict ourselves to IBB, in which only $\beta_{1}$ and $\beta_{4}$ are non-zero. This choice avoids introducing an explicit cosmological constant, which would make the entire bigravity model somewhat less appealing.

As shown in [35], IBB models have to satisfy $0<\beta_{4}<2 \beta_{1}$ in order to get an initial value of $r$ on the infinite branch. In particular, it was found that the best fit model occurs for $\beta_{1}=0.48$ and $\beta_{4}=0.94$ : from now on we refer to this choice as the reference IBB model. We then have

$$
\mathcal{H}^{2}=\frac{a^{2}\left(\beta_{1}+\beta_{4} r^{3}\right)}{3 r} .
$$

Here we derive the early time behaviour of the background evolution for later use. (corresponding to early time in IBB), Eq. (11) for IBB reduces to

$$
r^{\prime} \sim-\frac{3}{2}\left(1+w_{t o t}\right) r
$$

so that for $w_{t o t}=$ const (i.e., in radiation or matter dominated epochs) one has

$$
r \sim a^{-3\left(1+w_{t o t}\right) / 2}
$$

and the $f_{\mu \nu}$ scale factor $b(\tau)=r(\tau) a(\tau)$ goes as:

$$
b \sim a^{-\left(1+3 w_{t o t}\right) / 2} .
$$


The scale factor $b(\tau)$ therefore contracts instead of expanding as long as $w_{\text {tot }}>-1 / 3$. Moreover, in the same approximation,

$$
\mathcal{H}^{2} \approx \frac{a^{2} \beta_{4} r^{2}}{3}
$$

It is useful to derive an approximated estimate for the $b(\tau)$ bounce epoch. The bounce occurs $b^{\prime}=\left.0 \Leftrightarrow r^{\prime}\right|_{r_{b}}=-r_{b}$. If we assume a bounce after the radiation epoch, then the ratio at the bounce has to satisfy

$$
4 \beta_{1}-6 \beta_{1} r_{b}^{2}+\beta_{4} r_{b}^{3}=0
$$

From comparisons with observational data, we know that the best fit is close to $\beta_{4} \approx 2 \beta_{1}$ which leads to

$$
r_{b} \simeq 1+\sqrt{3}
$$

It is also useful, for future purposes, to take note of the approximate observational relation between $\beta_{1}$ and $\beta_{4}$ :

$$
\beta_{4}=\beta_{1}^{2} \frac{3\left(1-\Omega_{t o t 0}\right)-\beta_{1}^{2}}{\left(1-\Omega_{t o t 0}\right)^{3}}=\frac{3 \beta_{1}^{2}}{\left(\Omega_{t o t 0}-1\right)^{2}}+\mathcal{O}\left(\beta_{1}^{4}\right) ;
$$

This relation approximately corresponds to the degenerate line between $\beta_{1}$ and $\beta_{4}$ for a flat universe, when fitting data sets such as supernovae, CMB and baryonic acoustic oscillation data [35. Moreover, we require $\beta_{1} \lesssim 0.5$, to ensure that the solution of Eq. (16) at present time lies on the infinite branch.

\section{TENSOR PERTURBATIONS}

As we are interested on the effect of bigravity on gravitational waves, we now proceed with writing the tensor perturbation equations [31, 36, 39]. For the perturbed part of the metrics we adopt the transverse-traceless (TT) gauge, i.e. we select a transverse wave propagating along the $z$ direction. Then, the tensor metric perturbations are given by:

$$
h_{g(i j)}=\left(\begin{array}{ccc}
h_{g(+)} & h_{g(\times)} & 0 \\
h_{g(\times)} & -h_{g(+)} & 0 \\
0 & 0 & 0
\end{array}\right)
$$

and similarly for the tensor modes of the $f$ metric. We then obtain the following equations for both components (suppressing the subscripts,$+ \times$ )

$$
h_{n}^{\prime \prime}+\gamma_{n} h_{n}^{\prime}+\left(m_{n}^{2}+c_{n}^{2} \mathcal{H}^{-2} k^{2}\right) h_{n}=q_{n} h_{m}
$$

where the indices $n \neq m$ refer to $g$-metric and $f$-metric, respectively; we have then

$$
\begin{aligned}
\gamma_{g} & =2+\frac{\mathcal{H}^{\prime}}{\mathcal{H}}, & \gamma_{f} & =\frac{2 r^{2}+3 r^{2}+r\left(4 r^{\prime}-r^{\prime \prime}\right)}{r\left(r^{\prime}+r\right)}+\frac{\mathcal{H}^{\prime}}{\mathcal{H}} ; \\
m_{g}^{2} & =\mathcal{H}^{-2} a^{2} B r, & m_{f}^{2} & =\frac{\left(r^{\prime}+r\right)}{\mathcal{H}^{2} r^{2}} a^{2} B ; \\
c_{g}^{2} & =1, & c_{f}^{2} & =\frac{\left(r^{\prime}+r\right)^{2}}{r^{2}} ; \\
q_{g} & =\mathcal{H}^{-2} a^{2} B r, & q_{f} & =\frac{\left(r^{\prime}+r\right)}{\mathcal{H}^{2} r^{2}} a^{2} B,
\end{aligned}
$$

and where:

$$
B \equiv \beta_{1}+\beta_{3} r^{2}+r\left(2 \beta_{2}+\beta_{3} r^{\prime}\right)+\beta_{2} r^{\prime}
$$

These equations are equivalent to the ones in Refs. [31, 36]. In IBB (i.e. for $\beta_{0}=\beta_{2}=\beta_{3}=0$ ), $B$ is simply given by $\beta_{1}$. The coefficients $(27)-(30)$ for the two tensor equations are plotted in Fig 1 as a function of redshift, for the choice $\beta_{1}=0.48$ and $\beta_{2}=0.94$. For this reference model, when considering both matter and radiation, the bounce happens at a redshift $z_{b} \simeq 0.9$, with a corresponding $r_{b} \simeq 2.8$. 

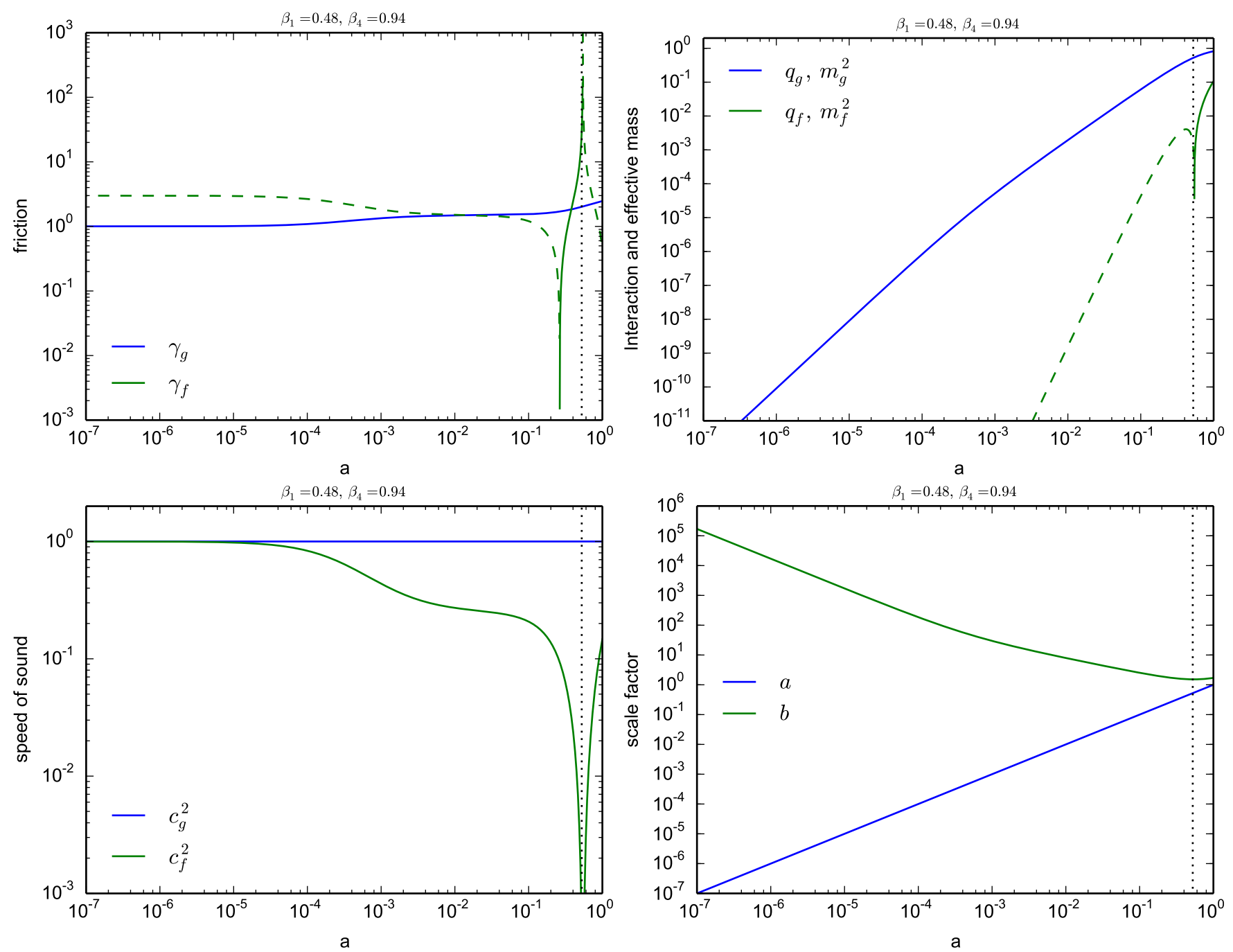

FIG. 1: Coefficients of the tensor equations for IBB 27,30). Solid/dashed lines indicate positive/negative values and the vertical dotted line marks the bounce of the reference metric. Note that the friction term in the $f$-metric is negative at early times, when the $f$-metric is contracting. Note also that the coupling and effective mass terms are equal, and the ones corresponding to the $f$ metric are very suppressed at early times. The bottom-right panel shows the evolution of the two scale factors.

Let us anticipate here an important feature of these equations. As it will be shown below (see also Ref. 39]), the equation for $h_{f}$ is unstable at early times since its friction term is negative as long as the scale factor $b(t)$ is collapsing instead of expanding. The fast growth of $h_{f}$ will then drive a fast growth of $h_{g}$ as well, through the coupling term. However, in the limit $r \rightarrow \infty$, the coupling coefficient $q_{g}$ becomes

$$
q_{g}=\frac{a^{2} r \beta_{1}}{\mathcal{H}^{2}} \stackrel{r \rightarrow \infty}{\longrightarrow} 3 \frac{\beta_{1}}{\beta_{4} r},
$$

and is therefore relatively small for large $r$. For the reference IBB model we have $\beta_{1} / \beta_{4} \approx 0.5$; more in general, according to Eq. 24), $\beta_{1} / \beta_{4} \approx 1 / 3 \beta_{1}$ to within factors of order unity, and therefore since $\beta_{1}<0.5$, we have the lower bound

$$
q_{g} \approx \frac{1}{\beta_{1} r} \geq \frac{1}{r}
$$

At recombination, for instance, we have $r \approx 10^{4}$ in IBB so that one needs a $h_{f}$ roughly $10^{4}$ times bigger than $h_{g}$ before the coupling term $q_{g} h_{f}$ becomes comparable to the $h_{g}$ terms in Eq. (26) and it starts driving the evolution of $h_{g}$. This means that, in principle, a growing mode in $h_{f}$ will take some time before affecting $h_{g}$. Whether this is enough to spoil the physical metric, is what we are going to test below. 
In the following subsections we discuss more in detail the time behavior of $h_{g}, h_{f}$ during the inflationary, radiation and matter eras.

\section{A. Inflation}

During a de Sitter epoch in which $H=$ const, one has $\mathcal{H}^{\prime} \sim \mathcal{H}$ and from Eq. [11:

$$
r \sim \text { const } .
$$

The tensor equations 26 then reduce to:

$$
\begin{aligned}
h_{g}^{\prime \prime}+3 h_{g}^{\prime}+h_{g}\left(\frac{k^{2}}{\mathcal{H}^{2}}+\frac{a^{2} \beta_{1} r}{\mathcal{H}^{2}}\right) & =\frac{a^{2} \beta_{1} r}{\mathcal{H}^{2}} h_{f}, \\
h_{f}^{\prime \prime}+3 h_{f}^{\prime}+h_{f}\left(\frac{k^{2}}{\mathcal{H}^{2}}+\frac{a^{2} \beta_{1}}{r \mathcal{H}^{2}}\right) & =\frac{a^{2} \beta_{1}}{r \mathcal{H}^{2}} h_{g}
\end{aligned}
$$

We can now assume $a^{2} \beta_{1} r \ll \mathcal{H}^{2}$ during inflation (ie $\rho_{\text {inf }} \gg \rho_{m g}$ ) so $h_{g}$ behaves as in GR. The same is true for $h_{f}$ since $a^{2} \beta_{1} / r \mathcal{H}^{2} \sim\left(\beta_{1} / \beta_{4}\right) r^{-3} \ll 1$. Since the inflationary equations are the standard ones, we expect the initial conditions to be unchanged and to apply equally well to $h_{g}$ and $h_{f}$.

\section{B. Radiation and Matter Dominated Era}

In the early time, we can approximate the ratio of scale factors as $r^{\prime}=-\frac{3}{2}\left(1+w_{t o t}\right) r$ which is solved by

$$
r=A a^{-\frac{3}{2}\left(1+w_{t o t}\right)},
$$

where $A$ is a suitable normalization constant of order unity. Furthermore we approximate $\mathcal{H}^{2} \simeq \frac{1}{3} \beta_{4} a^{2} r^{2}$. If the initial conditions for $h_{g}$ and $h_{f}$ are similar, then the source terms (30) are negligible at early times, i.e. small $a$, and the equations decouple. Furthermore, we find

$$
\begin{array}{rlrl}
\gamma_{g} & \simeq \frac{3}{2}\left(1-w_{t o t}\right), & \gamma_{f} & \simeq-\frac{3}{2}\left(3 w_{t o t}+1\right), \\
m_{g}^{2} & \simeq \frac{3 \beta_{1}}{A \beta_{4}} a^{\frac{3}{2}\left(w_{t o t}+1\right)}, & m_{f}^{2} \simeq-\frac{3 \beta_{1}\left(3 w_{t o t}+1\right)}{2 A^{3} \beta_{4}} a^{\frac{9}{2}\left(w_{t o t}+1\right)}, \\
c_{g}^{2} \simeq 1, & c_{f}^{2} \simeq \frac{\left(3 w_{t o t}+1\right)^{2}}{4} .
\end{array}
$$

Neglecting the mass term $m_{f}^{2}$ at early times, the tensor evolution for $f_{\mu \nu}$ is described by

$$
h_{f}^{\prime \prime}-\frac{3}{2}\left(3 w_{t o t}+1\right) h_{f}^{\prime}+\frac{h_{f} k^{2}\left(3 w_{t o t}+1\right)^{2}}{4 \mathcal{H}^{2}}=0 .
$$

At large scales the last term is negligible and one finds a growth of $h_{f}$ as $a^{3\left(3 w_{t o t}+1\right) / 2}$. Thus, when radiation dominates, $h_{f}$ increases very fast as $a^{3}$. Clearly, if one starts with $h_{f}^{\prime}=0$ then this growing mode is initially absent and it takes some time before it becomes visible. The evolution of $h_{g}$ has instead a constant mode $h \sim$ const until $h_{f}$ is large enough to source the growth of $h_{g}$, cf Eq. (32).

The early time approximation that leads to Eq (41) turns out to be a very good approximation also in the matter domination. In this regime $h_{f}$ increases as $a^{3 / 2}$ for super-horizon modes. When the coupling term becomes important, $h_{g}$ is driven by $h_{f}$ and acquires the same trend. Finally, when MDE ends and the system approaches a de Sitter behavior, the perturbations begin to decay.

For sub-horizon scales the behavior is influenced by the $h_{f}$ time-dependent sound speed. An asymptotic form for large $k$ can however be found. In this regime we can neglect the mass and the coupling terms, and the $h_{g}, h_{f}$ equations during either RDE or MDE have the general form

$$
h_{n}^{\prime \prime}+\gamma_{n} h_{n}^{\prime}+\beta_{n} k^{2} a^{\eta} h_{n}=0,
$$



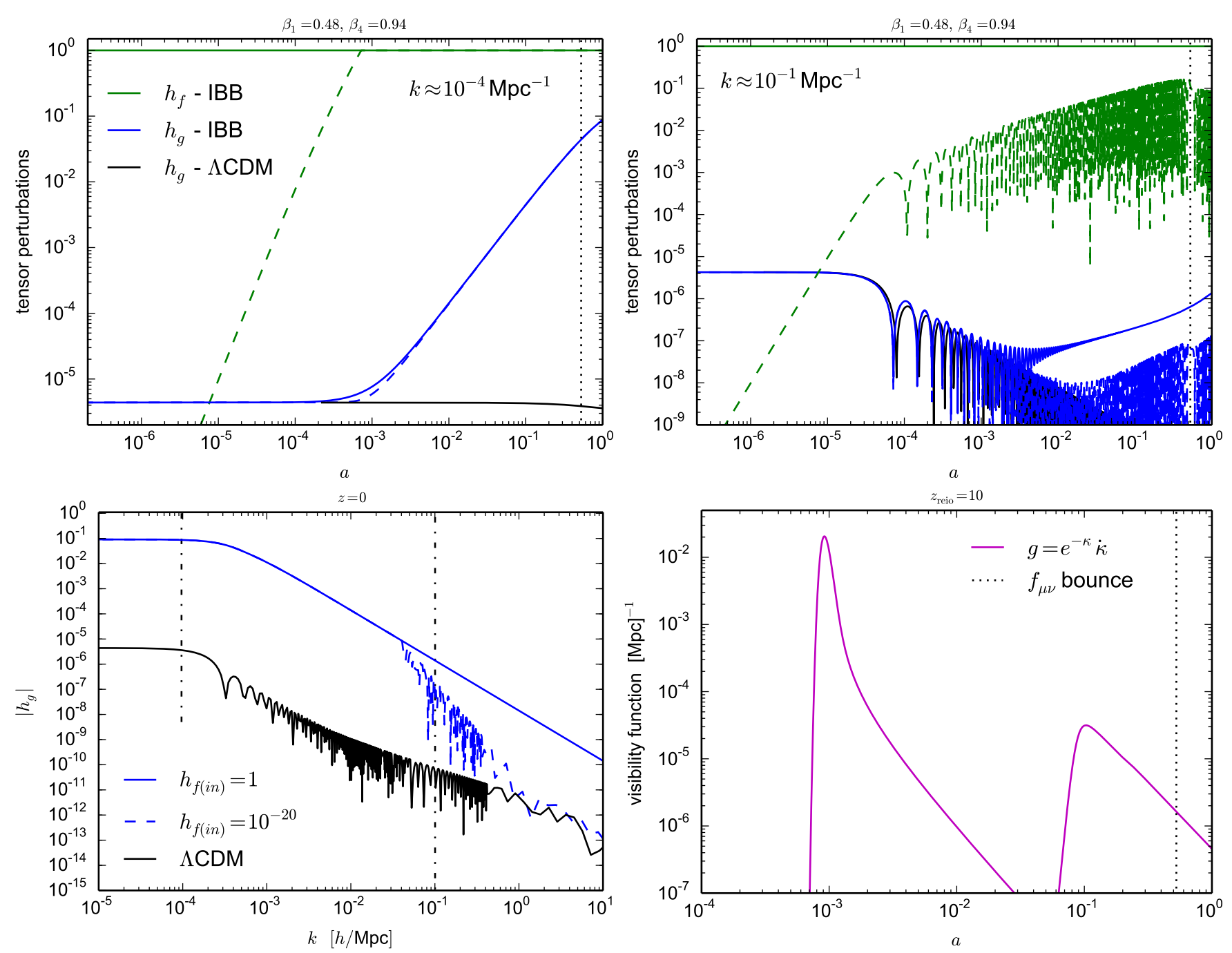

FIG. 2: Evolution of tensor perturbations at different scales (top panels) and scale dependence of tensor perturbations at $z=0$ (bottom left panel). All scales include a cut-off when non-linearity is reached; $h_{g}$ does not reach the cut-off for any scale and redshift. The initial conditions have been chosen so that $h_{f}=1$ is initially non-linear (solid lines) or starts at a small value (dashed lines) and becomes non-linear at latter times. The latter choice corresponds to $h_{f(i n)}^{\prime} \approx h_{f(i n)}=10^{-20}$ at $a=10^{-10}$ (see section $\mathrm{VA}$. The two modes shown in the top panel correspond to the vertical dash-dotted lines in the bottom left panel. For reference, we recall the standard CMB photon visibility function (bottom right panel), whose peaks correspond to recombination and reionization epochs (see section IV]. The bounce of the reference metric has been indicated with a dotted vertical line.

where the index $n$ stands for $g, f$ and $\eta=1+3 w_{t o t}$ and $\beta_{n}$ is an irrelevant constant. The general solution can be easily written in terms of the Bessel functions but here we need only the asymptotic behavior for large $k$ or late times, which is

$$
h_{n} \sim a^{-\left(\frac{\gamma_{n}}{2}+\frac{\eta}{4}\right)}
$$

times fast oscillations. We see then that for sub-horizon modes $h_{f}$ grows as $a^{1}$ in RDE, as $a^{1 / 2}$ in MDE and a final decay as $a^{-1}$ when approaching the future deSitter phase, while $h_{g}$ decays as in the standard case as $a^{-1}$ in all eras (before being driven to growth by the coupling to $h_{f}$ ). For very large wavenumbers the coupling and the mass terms are ineffective at all times and the $h_{g}$ equation reduces to the standard case. This implies that there is no large effect to be expected for the directly detectable range of gravitational waves, which is around $0.1 \mathrm{~Hz}$ or $k \approx 10^{14} \mathrm{Mpc}^{-1}$ (see e.g. [43]), although a precise calculation is beyond the scope of this paper. 


\section{Inflationary initial conditions}

We can now use the $a^{3}$ growth mode during radiation to estimate the order of magnitude effect of the tensor modes at recombination (a more precise estimation will be obtained numerically in sections IV, V). Inflation ends at some energy scale that can vary from $10^{15} \mathrm{GeV}$ to few $\mathrm{MeV}$ depending on the model. The upper limit comes from the bounds on the amplitude of tensor perturbations, indicating that the energy scale of inflation is at least that of Grand Unified Theories when observable modes are produced. The lower bound is inferred from the need of a radiation dominated universe in thermal equilibrium during big bang nucleosynthesis. These values are reached when the scale factor was $a_{\text {inf }} \approx 10^{-9}$ at the latest. Since super-horizon tensor modes grow as $a^{3}$ during radiation domination, in the most favourable case of inflation ending just before big bang nucleosynthesis one would obtain an enhancement until recombination $a_{\text {rec }} \approx 10^{-3}$ of $h_{f(\mathrm{rec})} \sim 10^{18} h_{f(e)}$ roughly, where the subscript $e$ denotes the end of inflation. If $h_{f(e)}$ has the standard value approximately equal to $H_{e} / T_{P} \approx T_{e}^{2} / T_{P}^{2}$ during inflation, where $T_{P} \approx 2.4 \cdot 10^{18} \mathrm{GeV}$ is the reduced Planck temperature/energy and $T_{e}$ is the inflationary energy scale (here for simplicity assumed to be similar to the energy at the end of inflation), then the value at recombination of $h_{f}$ for a wave that reenters horizon at recombination or larger is roughly

$$
h_{f(\mathrm{rec})} \approx\left(\frac{a_{\mathrm{rec}}}{a_{e}}\right)^{3} h_{f(e)}=\left(\frac{T_{e}}{T_{\mathrm{rec}}}\right)^{3}\left(\frac{T_{e}}{T_{P}}\right)^{2}
$$

Shorter waves reenter before and therefore grow less. If this value has to be compatible with the level of fluctuations in the CMB polarization spectra, then it should be lower than about one tenth of the temperature fluctuations; we take conservatively the level $10^{-7}$. The same value should be taken for $h_{f}^{\prime}$ since inflation excites both the tensor mode and its momentum conjugate. However we do not detect directly $h_{f}$ but rather the $g$-metric mode $h_{g}$ which is coupled to matter, so as already noticed one can have a value of $h_{f}$ larger than $h_{g}$ by a factor of $q_{g}^{-1} \approx 10^{4}$ at recombination. Putting therefore $h_{f(\mathrm{rec})} \leq 10^{-3}$, we obtain an upper limit to the temperature at the end of inflation $T_{e} \approx 10 \mathrm{GeV}$. Since tensor modes impact CMB also at reionization, when the coupling term $q_{g}$ is closer to unity, this limit should be lowered to roughly

$$
T_{e} \approx 1 \mathrm{GeV}
$$

(a similar limit has been obtained also in [39]). It might be interesting to remark that the superhorizon growing mode breaks the standard link between tensor modes and inflationary scale due to the presence of the coupling: now in principle one can have observable tensor modes even in low scale inflation.

Any inflationary model with higher energy scale will generate excessive power on the tensor modes unless the inflationary initial conditions are suppressed with respect to the standard value or their growth is reduced. Taken at face value, this shows that the $a^{3}$ growing mode can be reconciled with observations only in the rather extreme scenarios of very low-energy inflation, as e.g. in the models discussed in Ref. (44-46]). Fixing the initial conditions to a more conservative era for the end of inflation, e.g. $T \sim 10^{3} \mathrm{GeV}$, would produce the huge value $h_{f(\text { rec })} \sim 10^{39} h_{f(e)} \sim 10^{7}$.

Barring the case of very low-energy inflation, then, the IBB model is at odds with CMB observations. In the rest of the paper we will explore more or less contrived ways to overcome this difficulty.

\section{The non-linear cut-off}

The tensor perturbations in the reference metric grow so fast that they will eventually become non-linear. At this point, the perturbative treatment followed so far breaks down and one has to take into account higher order corrections, or even the full equations of motion in order to correctly reproduce the dynamics. A natural question is then what happens to the two metrics after non-linearity is reached: the evolution would then need to be calculated self-consistently in a non-linear theory for bigravity, which is beyond the scope of this paper. This problem is not new to Dark Energy models. There are cases such as growing neutrino cosmologies [47] in which the effect of non-linearities becomes important and needs to be taken into account also when dealing with the CMB predictions [48. In that scenario, the fast growth actually leads to stable non-linear structures (which are a way to test the model rather than an argument to exclude it based on linear theory).

Following the idea of Ref. [48], we then stop the evolution of perturbations at some cut off amplitude value $h^{\text {cut }} \approx 1$, when non-linearity is approximately reached. This prescription is applied to both $h_{g}$ and $h_{f}$ and has the effect to partially stop the 'dragging' of the second metric $h_{f}$ over the standard one $h_{g}$. Such assumption is adopted here for simplicity, as for now our interest is to give a consistent estimate of how big is the impact of the growing mode on CMB spectra and tensor perturbations when non-linearity is reached. 
In practice, since the growth of $h_{f}$ is very rapid in the early Universe, this is equivalent to fixing $h_{f}=h^{\text {cut }}=1$ from the very beginning, with the consequence that the perturbations of the reference metric are not dynamical anymore. Nevertheless they still affect the tensor modes of the physical metric due to the coupling (32). The overall evolution as a function of the scale factor for two values of $k$ and two different choices of the initial conditions is plotted in Fig, 2 for both $h_{g}$ and $h_{f}$, once the bound has been applied. Figure 2 also shows a similar behavior between a model in which $h_{f}$ starts saturated and one in which the cutoff value is reached during the evolution. It is also shown how, even though the cutoff is applied to both metrics, $h_{g}$ never reaches it during its evolution. Note that the full non-linear dynamics might produce other effects. For example, in the limit of scales smaller than the horizon, one finds the oscillating behavior in e-folding time $h_{g}, h_{f} \sim e^{i m N}$ with eigenfrequencies:

$$
m= \pm \frac{r+r^{\prime}}{\mathcal{H} r} .
$$

This oscillating behavior is then always present in the sub-horizon solutions, overimposed to an amplitude modulation, as shown for the smallest scale in Fig. 2 (top right panel). In this case, setting $h_{f}$ to the non-linear cut-off value leads to a growing behavior plus a damping of the initial oscillations. On the other hand, the model with fine tuned initial conditions displays the oscillatory behavior expected from linear theory $(46)$, and in this case the non-linear value $h_{f} \sim 1$ is not reached, at least for Fourier modes corresponding to small scales. In this case the negative friction of the reference metric gets compensated by the positive friction from the physical metric. Nonetheless we expect our method to give a correct quantitative estimate of the observable effects of the growing mode on the CMB.

\section{COSMIC MICROWAVE BACKGROUND ANISOTROPIES IN BIGRAVITY}

The Cosmic Microwave Background (CMB) was shown to be a powerful probe to test not only early time cosmology but also Dark Energy and Modified Gravity models [49. In particular, in this paper we are interested in the effect that tensor perturbations in bigravity have on the CMB power spectra. At recombination, when photons are not anymore tightly coupled to baryons but decoupling has not occurred yet, electrons can be scattered simultaneously by photons coming from cold and hot spots. In presence of a quadrupole temperature anisotropy, the scattered photons will be linearly polarized and the CMB radiation will be characterized not only by its intensity, but also by its polarization. CMB polarization can be expressed in a tensor normal basis in Fourier space, in terms of $\mathrm{E}$ and B modes. While scalar perturbations can only produce an E mode (primordial) polarization pattern, tensor perturbations can feed both $\mathrm{E}$ and $\mathrm{B}$ primordial modes. Therefore any change in the evolution of tensor perturbations predicted in bigravity will affect the polarization spectra.

At later times, polarization and temperature anisotropies are further modified during reionization. Reionization occurs at a much lower redshift, when the universe becomes partially ionized due to the formation of the first stars, allowing CMB photons to partially rescatter. The recombination and reionization eras correspond to peaks in the visibility function shown in figure 2. The visibility function $g(t)=\exp (-\kappa) \dot{\kappa}$ (where $\kappa$ is the optical depth and $\dot{\kappa}$ is its derivative with respect to conformal time $\tau$ ) gives the probability that a photon last scattered in the conformal time interval $[\tau, \tau+d \tau]$. Due to the importance of the coupling at relatively low redshift, the most important effects of tensor modes on the CMB are imprinted during the reionization epoch.

In the following, we have only modified tensor perturbations, assuming that the contribution of scalar perturbation is small enough to be neglected, as scalar modes affect B mode polarization only indirectly, via lensing of $\mathrm{E}$ modes, at scales $\ell \gtrsim 150$. Of course, if polarization is large enough, it might also feed back the scalar spectra. However, this seems a good enough first approximation to test the specific effect of the growing mode on the BB spectra. We implemented the tensor evolution equations in two publicly available Boltzmann codes, CAMB [50] and CLASS [51], and compared the results obtained in the various cases to verify their mutual consistency.

As discussed in the previous section, our aim is to check the effect on the CMB spectra consistently, i.e. taking into account that, by definition, we cannot trust any result derived assuming linear perturbation theory when perturbations become non-linear. We then fix $h_{f}=h^{c u t}=1$ from the beginning and evolve only the $g$-metric tensor $h_{g}$, for which we will assume standard initial conditions:

$$
A_{t}=r_{T / S} A_{s}\left(\frac{k}{k_{p}}\right)^{n_{T}}
$$

with a fiducial tensor-to-scalar ratio $r_{T / S}=0.05$, a scalar amplitude $A_{s}=2.21 \times 10^{-9}$ and a tensor spectral index given by the self-consistency condition of single field slow-roll inflation $n_{T}=-\left(2-r_{T / S} / 8-n_{s}\right) r_{T / S} / 8$, where the fiducial scalar spectral index is $n_{s}=0.9645$ (in section $\mathrm{VA}$ we explore the effects of changing the IC on the tensor modes.). For bigravity we choose the best fit model $\beta_{1}=0.48, \beta_{4}=0.94$ with $\Omega_{c d m}=0.13, \Omega_{b}=0.05$, while for 

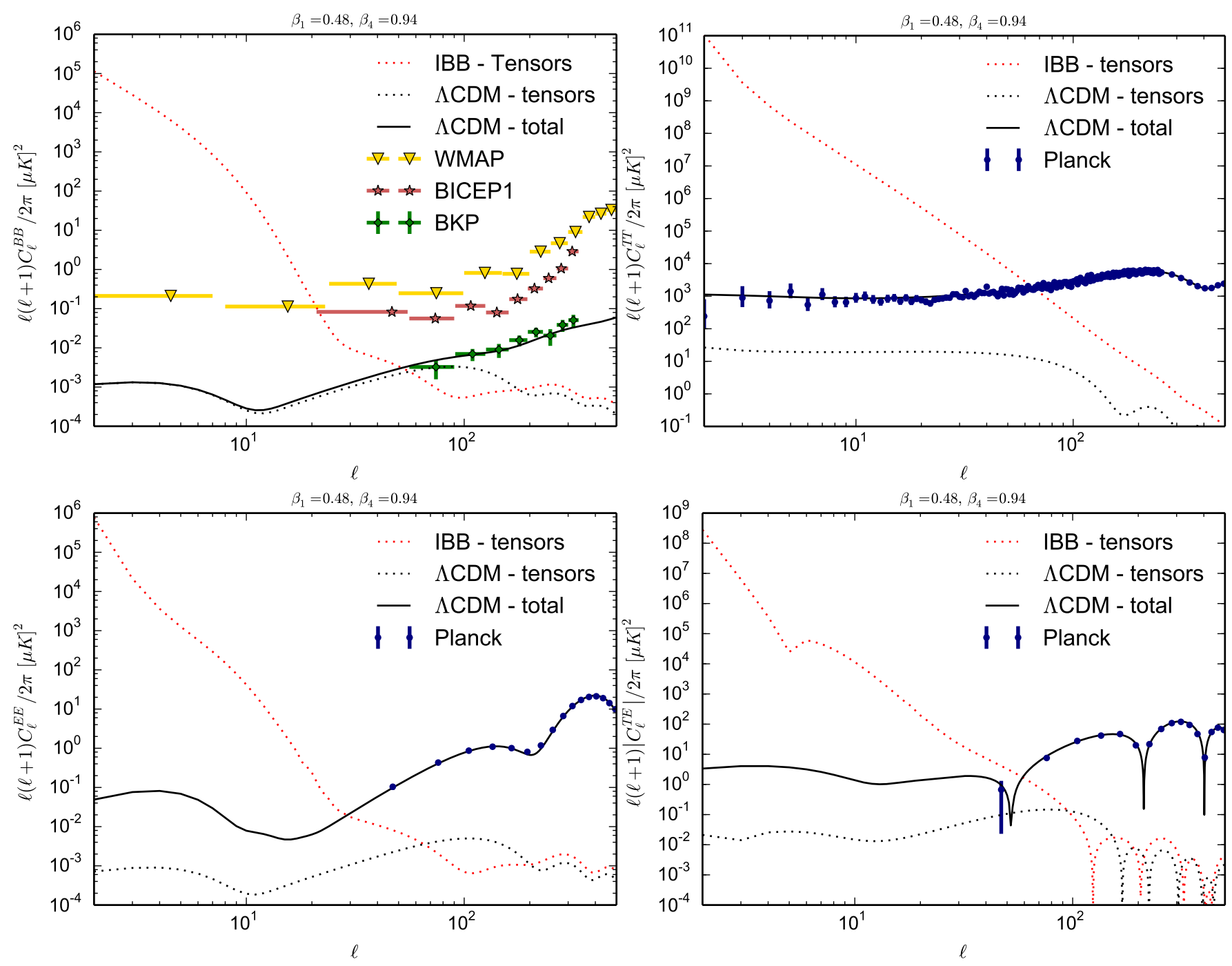

FIG. 3: Effects of the growing mode in the $f$-tensors on the CMB. All the plots assume IBB with $\beta_{1}=0.48, \beta_{4}=0.94$, $\Omega_{m}=0.18$ with $\Omega_{b}=0.05, h=0.67, r_{T / S}=0.05$ and the spectral index determined by inflationary self-consistency conditions. The tensor perturbations have been assumed to start at non-linear cutoff value $h_{f}=1$.

the reference $\Lambda \mathrm{CDM}$ model we choose Planck $2015 \mathrm{TT}$, EE, TE + lowP marginalized values [52], i.e. $\Omega_{c d m}=0.26$, $\Omega_{b}=0.05$. In both bigravity and $\Lambda \mathrm{CDM}$ cases the fiducial optical depth is $\tau=0.079$, corresponding to $z_{\text {reio }}=10$. In order to test the IBB model, we compare the achieved spectra with up to date CMB observations, using Planck 2015 data [53] for TT, TE and EE spectra, while for the BB spectrum we rely on WMAP [54] and BICEP1 [55] together with the joint BICEP2, Keck, Planck analysis [56]. Figure 3 shows the tensor contribution to the CMB temperature, polarization and cross spectra for the fiducial bigravity model described above. Large angular scales are the most sensitive to the growing modes in the $f$-metric, because the coupling to the physical metric is only important at low redshifts, after recombination. On these scales the tensor perturbations give contributions to the power spectra that are too large to be compatible with CMB data. Even for the T and E polarization spectra, the tensor contribution in IBB bigravity overshoots the observed values by several orders of magnitude for $\ell \lesssim 60$. Since both the scalar and tensor contributions to TT and EE spectra are positive definite, it is impossible that a reduced scalar contribution compensates the (large) tensor part in order to fit the data. The conclusion is that the cut-off on the growing mode is not enough to save the model. 

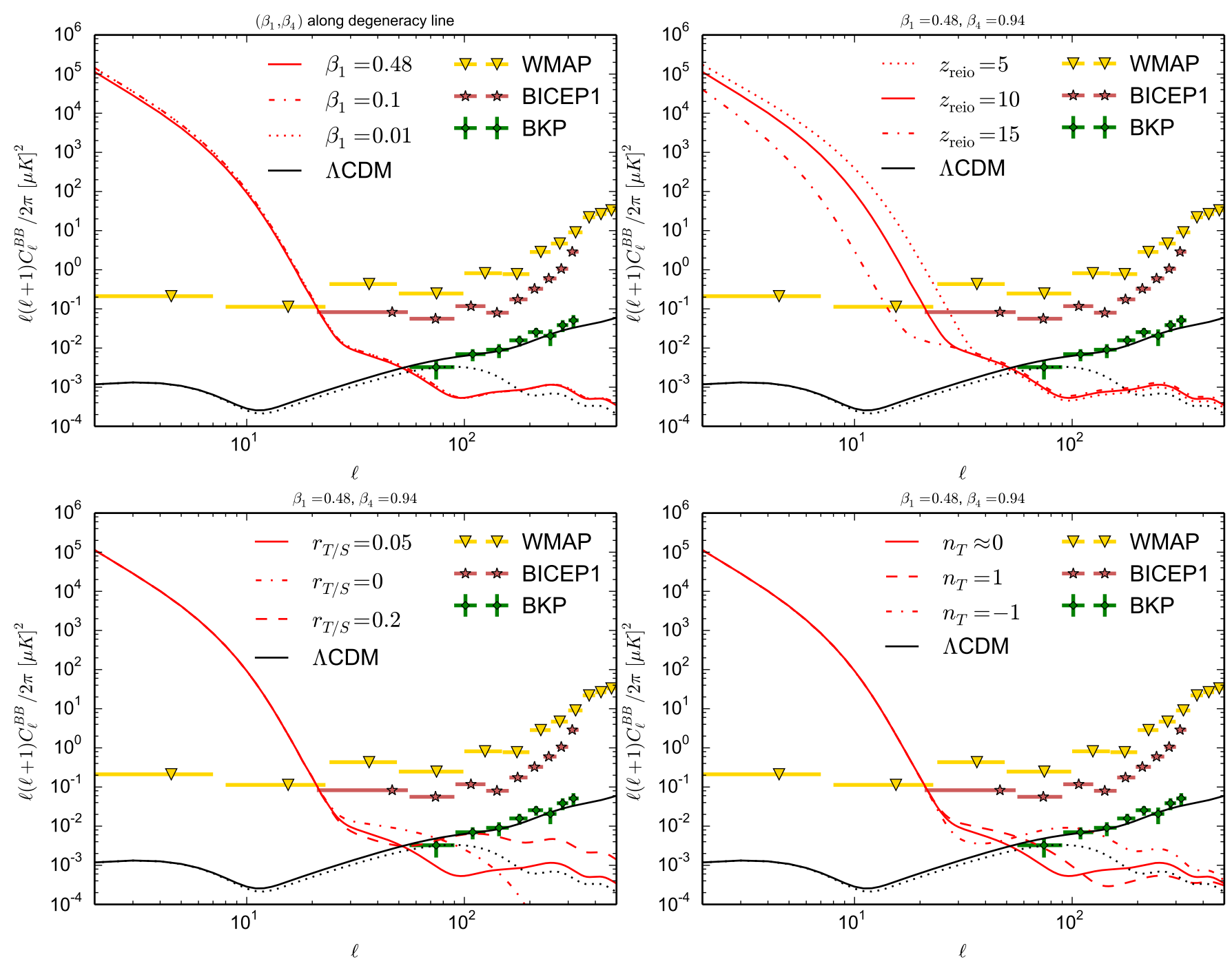

FIG. 4: Non-solutions to the problem of growing modes in the reference metric: varying the IBB parameters across the degeneracy line (top left panel), the reionization redshift (top right) and the initial conditions for tensor perturbations in the physical metric (bottom panels). The remaining model details are the same as in Fig. 3 . In particular, the reference metric perturbations are set initially to the non-linear cutoff value. All solid lines correspond to the standard values described in section IV

\section{POSSIBLE SOLUTIONS TO THE GROWING MODE PROBLEM}

A first attempt to overcome the growing mode problem is to verify how much the effect on the spectra depends on the choice of the fiducial model. We investigated the effect of changing the bigravity parameters $\beta_{1}$ and $\beta_{4}$, choosing them close to the degeneracy curve $(24)$, the redshift of reionization $z_{\text {reio }}$, the tensor to scalar ratio $r_{T / S}$ and the tensor spectral index $n_{T}$ characterizing the shape of the primordial power spectrum for tensor perturbations. These effects are shown in Fig. 4, showing that simple variations of these cosmological parameters do not offer a sufficient improvement.

The observable impact on the CMB is produced in the reionization era because the coupling between the physical and reference metric's tensor perturbations is only relevant at low redshift. Shifting $z_{\text {reio }}$ in the range $(5,15)$ has only a small effect on the BB spectrum. Further changes would spoil the predictions for EE and TE spectra and enter in contradiction with the Gunn-Peterson limit [57]. Higher reionization redshifts reduce the tension slightly, mainly because the perturbations in $h_{g}$ are smaller at earlier times and on smaller scales. Another attempt that does not work is to modify the initial conditions for perturbations of the physical metric. Varying the spectral index and the tensor to scalar ratio only has an impact on relatively high multipoles $\ell>30$, on which the tensor perturbations are predominantly imprinted during recombination. For lower multipoles the evolution of $h_{g}$ is dominated by the coupling 
to $h_{f}$, which overshoots the initial conditions on large scales due to its large value.

Concordance with observations can neither be achieved by varying the IBB parameters $\beta_{1}, \beta_{4}$. The fast growth of the $h_{f}$ tensor perturbations leads to growing $h_{g}$ tensors due to the coupling in 26 which is proportional to $\beta_{1} r / \mathcal{H}^{2}$. One might expect that a change in the betas could lead to higher values of $r$ and, thus, a suppression of the coupling at early times (note that $\mathcal{H}^{2} \propto r^{2}$ in RDE). Higher values of $r$ are possible when lowering $\beta_{1}$ since $r_{0}=\left(1-\Omega_{\text {tot } 0}\right) / \beta_{1}$, where $r_{0}$ denotes the present value of $r$ and one has $r>r_{0}$ during the entire evolution. However, in order to fit observations we have to choose parameters being close to the degeneracy curve 24 , i.e. $\beta_{4} / \beta_{1}^{2} \simeq$ const. As already mentioned, at early times the coupling term is then proportional to $q_{g} \approx 1 / \beta_{1} r$ (see Eq. 33). A smaller coupling would of course help in delaying or reducing the effect of the $h_{f}$ growing mode on $h_{g}$. Since the evolution for large $r$ is nearly independent of the coupling parameters (see Eq. 18$)$, and since $\beta_{1} \lesssim 0.5$, the ratio $1 /\left(\beta_{1} r\right)$ cannot decrease much below the reference case. Figure 4 shows clearly that the effect of varying $\beta$ 's is negligible, at least when the coefficients remain along the degeneracy line.

One loophole in the line of argument above is to leave the observational degeneracy curve (24) and add an explicit cosmological constant $\beta_{0}$. This transforms the IBB model into a form of $\Lambda$ CDM plus a small admixture of massive bigravity. The coupling coefficient $q_{g}$ does not explicitly depend on $\beta_{0}$ and reduces to:

$$
q_{g}=\frac{3 \beta_{1} r^{2}}{\beta_{1}+\beta_{4} r^{3}}
$$

From the $f_{\mu \nu}$-Friedmann eq. 16 we find that $r$ scales like $\beta_{4}^{-1 / 2}$ for small values of $\beta_{1}$ which leads to:

$$
q_{g} \simeq \frac{9 \beta_{1}}{\beta_{1} \beta_{4}+3 \sqrt{3 \beta_{4}}}
$$

Thus, one is able to get an arbitrarily small coupling when choosing values of $\beta_{1}$ that are sufficiently smaller than $\beta_{4}$. In this regime, the massless graviton dominates over the massive one and the cosmological evolution tends to that of $\Lambda \mathrm{CDM}$. It is clear that such a model is not particularly interesting from a cosmological point of view because hardly distinguishable from the standard model and therefore here we will not investigate it further.

In order to render the model viable without adding a cosmological constant, it is necessary to adopt a more radical solution. In the following subsections we explore how IBB can be reconciled with observations by fine-tuning the initial conditions in the reference metric perturbations, lowering the non-linear cut-off or modifying the theory.

\section{A. Changing the initial conditions for $h_{f}$}

Our next attempt consists in checking whether fine tuning the initial conditions can compensate the effect of the growing mode on the CMB spectra, as illustrated in Fig. (5). We specify the initial conditions in terms of the growing solution in the radiation era

$$
h_{f}(a)=h_{f(\mathrm{in})}\left(a / a_{\text {in }}\right)^{3}, \quad h_{f}^{\prime}=3 h_{f}(a),
$$

found in section III B $^{1}$ We find that the initial conditions have to be fine tuned to zero to at least the level of one part in $10^{26}$ at $z_{\text {in }}=10^{10}$ in order to fit current limits on the BB spectrum. This choice of the initial epoch corresponds to an era before Big Bang Nucleosynthesis, which as already mentioned is a hard lower bound for the end of inflation. One can easily relate to earlier times in order to specify the IC at the reheating epoch, when inflation ends and tensor perturbations start growing. Table I extrapolates the result to the range of energies in which inflation might have ended.

\footnotetext{
${ }^{1}$ One can in general fix $h_{f}$ and $h_{f}^{\prime}$ independently for each wavenumber, but we restrict to the simpler choice 50 here. If more general IC are considered, a necessary condition for the growing mode to be sufficiently suppressed is that the time derivative is small. This condition is sufficient as long as $h_{f(\text { in) }}$ is well below the cutoff value (see next subsection).
} 

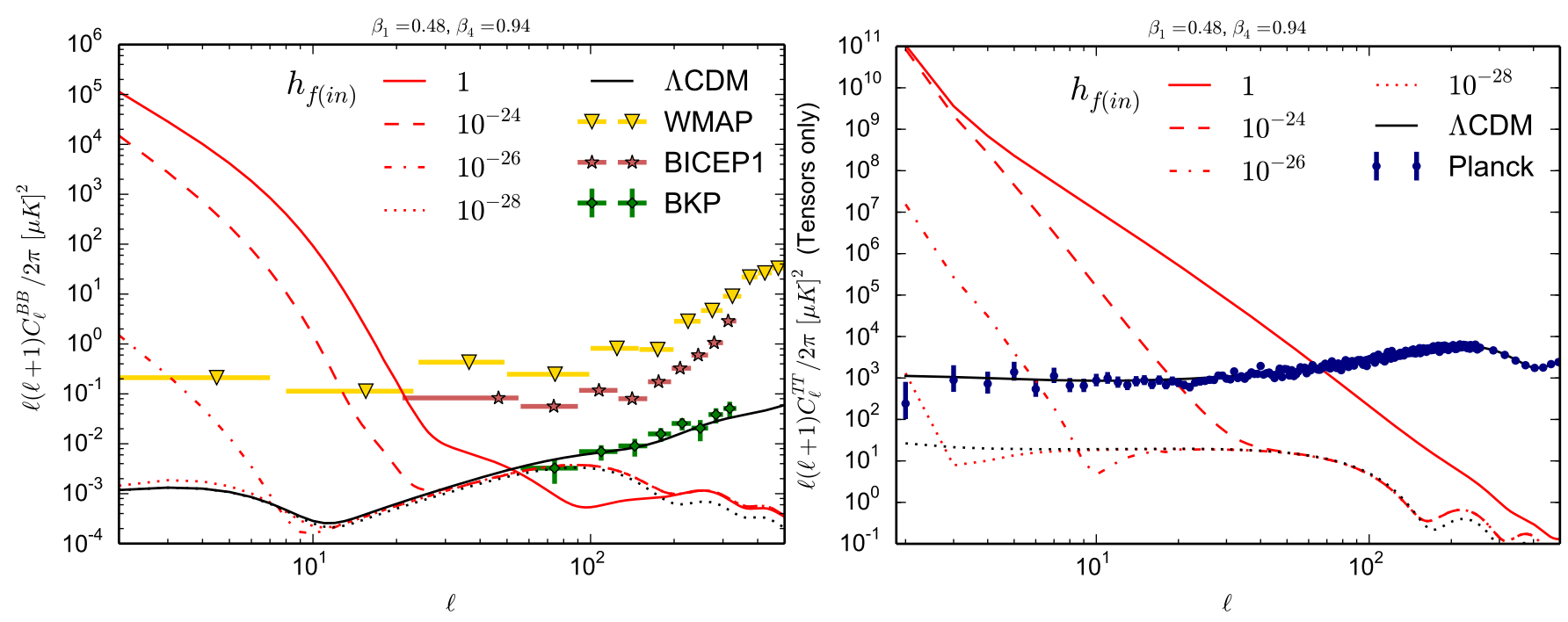

FIG. 5: BB (left panel) and TT (right panel) spectra using fine-tuned initial conditions as described in section V A The initial amplitude and time derivative have been specified according to the growing solution 50 at a fiducial scale factor $a_{\text {in }}=10^{-10}$. Bigravity BB spectra (red lines) only contain the primordial tensor contribution, while $\Lambda$ CDM spectra (black solid lines) includes the contribution of both scalar and tensor perturbations. The evolution of tensor perturbations has been stopped whenever $h_{g, f}=1$ is reached (cf. section III D).

\begin{tabular}{lcccc} 
& BBN & fiducial & $1 \mathrm{GeV}$ & GUT \\
\hline Bounds on $h_{f(\text { in })}$ & $\lesssim 10^{-19}$ & $\lesssim 10^{-25}$ & $\lesssim 10^{-31}$ & $\lesssim 10^{-82}$ \\
Scale factor $a$ & $\sim 10^{-8}$ & $\sim 10^{-10}$ & $\sim 10^{-12}$ & $\sim 10^{-29}$ \\
Temperature $T$ & $\sim 0.1 \mathrm{MeV}$ & $\sim 10 \mathrm{MeV}$ & $1 \mathrm{GeV}$ & $\sim 10^{16} \mathrm{Gev}$ \\
\hline
\end{tabular}

TABLE I: Upper bounds on $h_{f}$ extrapolated to different epochs using the growing mode, eq. (50). The limits shown are based on the results for BB spectra, which only depend on the tensor sector at low multipoles where the theory enters in tension with the data. Considering TT spectra would tighten these bounds, as can be inferred from the left panel of figure 5 .

As already noticed, the only way to generate naturally a very low level of tensor modes compatible with CMB without any cut-off is to assume that inflation ends at an energy scale not larger than $1 \mathrm{GeV}$. If however one assumes the non-linear cut-off, then the inflation energy scale bound can be relaxed. From Table I we see empirically that the initial condition for $h_{f}$ is related to the end of inflation energy scale $T_{e}$ (expressed in GeV) as $h_{f(\mathrm{in})} \approx 10^{-31} T_{e}^{-3}$. Then one has

$$
h_{f(\mathrm{in})} \approx\left(\frac{T_{e}}{T_{P}}\right)^{2} \approx 10^{-31}\left(\frac{1 G e V}{T_{e}}\right)^{3}
$$

or $T_{e} \approx 25 \mathrm{GeV}$, a more realistic scale range for low-energy inflation.

A sufficiently small value of $h_{f}^{\prime}$ might also be provided by a more exotic inflationary mechanism. During inflation no growing modes occur on the perturbations of the $f$ metric. Some solutions, such as increasing the mass of the graviton at very early times, might naturally generate the low values needed to reconcile the model with observations (the problem of growing classical perturbations is common to ekpyrotic scenarios alternative to inflation [58]). Outside of these rather unconventional, although not impossible, cases, the conclusion we draw is that only very fine tuned initial conditions allow to reconcile bigravity with CMB observations.

Even if inflation ends at a sufficiently low scale or a mechanism to suppress $h_{f(\mathrm{in})}^{\prime}$ exists, it has been argued by Cusin et al. [39] that non-linear corrections would spoil the small value of $h_{f}^{\prime}$. Although we will not investigate this issue further here, we note that the nature of the theory might protect the tensor modes against such terms. This is precisely what happens in the linear equation (26), in which the source term is highly suppressed. If a similar suppression occurs also on the non-linear source terms, the fine tuned initial conditions can render the model compatible with CMB observations (assuming that there are no additional complications in the scalar sector). 

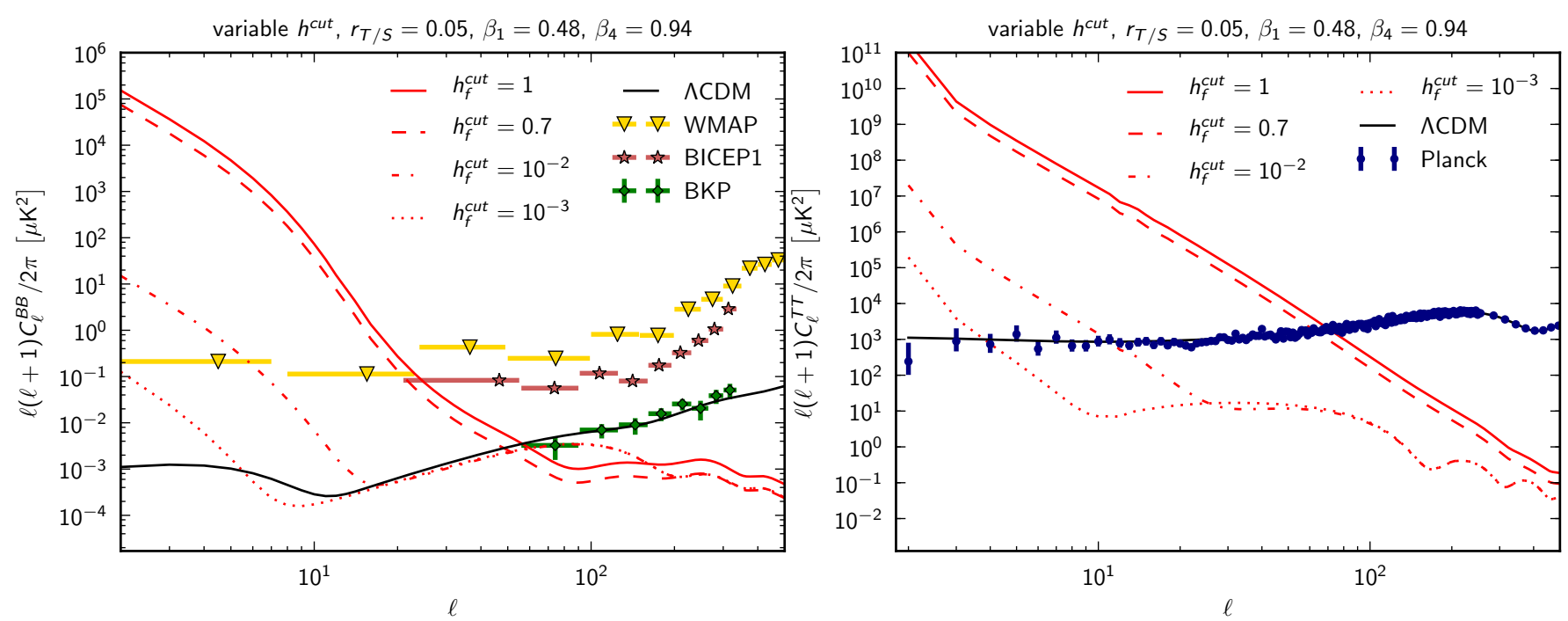

FIG. 6: BB (left panel) and TT (right panel) using a varying cutoff $h^{\text {cut }}$. The evolution of $h_{f}$ and $h_{g}$ is frozen when those reach, respectively, the cutoff value $h^{\text {cut }}$. Bigravity BB spectra (red lines) only contain the primordial tensor contribution, while $\Lambda$ CDM spectra (black solid lines) and observational data points contains the contribution of both scalar and tensor perturbation.

\section{B. Lowering the cut-off}

Another possible solution to mitigate the effect of IBB on CMB spectra is to assume that non linear effects begin to be not negligible before $h_{f}$ reaches unity; this can be thought as an effective way to treat the impact of non linear effects which, even if dominant when $h_{f}$ is above unity, can start to affect the evolution of the perturbations even for lower values. Therefore, in Figure 6 we show the behavior of TT and BB spectra for different values of $h^{\text {cut }}$ at which we freeze the evolution of the metric perturbations. One would need to suppress the cutoff scale by at least three orders of magnitude to reconcile theoretical BB spectra with currently available limits and possibly an even lower value to make the TT spectrum acceptable for current data, once the scalar contribution is taken into account.

It seems contrived that non-linear effects might play a role at such small values of the cutoff. However, theories of massive gravity are known for having strong non-linear effects in certain limits, such as the Vainshtein mechanism [59, 60. As long as numerical results for the non-linear evolution of tensor perturbations are not available, we must contemplate the possibility that the cut-off could be lower than unity and even significantly lower.

\section{Modifying the theory}

The growing modes in the reference metric could possibly be reconciled with CMB data by a suitable modification of the theory. Here we explore a phenomenological modification in which a redshift dependence of the $\beta_{i}$ parameters is assumed; this kind of behavior might be achieved in generalized massive gravity models [61, where a time dependence of the mass parameters is introduced without the addition of any new dynamical degree of freedom. Our modification consists on setting $\beta_{1}=\beta_{4}=0$ until a certain switch redshift $z_{s}$ is reached. Then bigravity becomes active and the evolution described in Section III is switched on. The considered background evolution instead is the one produced by bygravity at all redshift, as IBB well approximates the standard background, which should take place at $z>z_{s}$, at early times.

The results in Figure 7 show how switching on bigravity at approximately the redshift of matter-radiation equality $\left(z_{s} \approx 10^{3}\right)$ can produce an acceptable BB spectra when comparing with current data. In the TT case instead, the contribution of scalar modes to the spectrum can possibly lead to the necessity of an even lower value of $z_{s}$.

There are additional ways in which the theory might be modified while retaining the original field content of bigravity. In the following we will describe these possibilities, although addressing them in detail will be left for future work. A possible modification is to allow for branches different than IBB. So far, only branches in which $r$ evolves from $r=0$ or $r \rightarrow \infty$ were considered. In [29] all remaining cases due to a non-viable behavior were excluded. However, some of those conditions based on expectations of a standard cosmological evolution, like an expansion at all times and the existence of a matter/radiation dominated era in the asymptotic past. It might be interesting to study these 

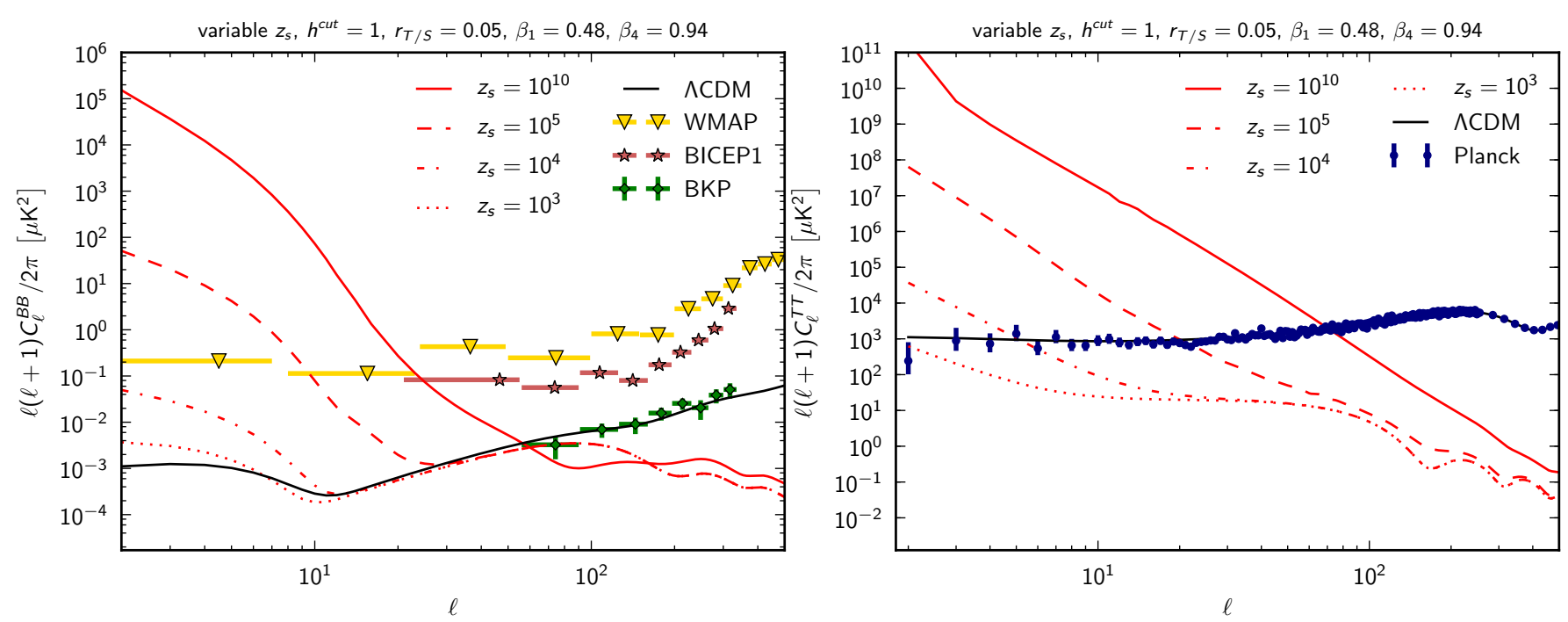

FIG. 7: BB (left panel) and TT (right panel) using a varying initial redshift $z_{s}$. If $z>z_{s} h_{f}$ follows the same standard equation as $h_{g}$, while for $z \leq z_{s}$ the evolution described in Section III is switched on. Bigravity BB spectra (red lines) only contain the primordial tensor contribution, while $\Lambda$ CDM spectra (black solid lines) and observational data points contains the contribution of both scalar and tensor perturbation.

disregarded branches. Additionally, both metrics are usually assumed to be FLRW at background level, even though this is assumed only for simplicity. In Ref. [62] the authors considered more general types of metrics which might lead to interesting evolutions at background and linear level and could also have an impact on the tensor evolution.

An additional possibility is to modify the coupling to matter. Even though both metrics are a priori equally footed, we let only one of them couple to matter while the remaining metric stays unobservable. An additional coupling of the reference metric to matter would influence the tensor perturbation and might be able to tame the fast growth (see e.g. Refs. 63, 64 for further discussion on bi-metric couplings). An additional coupling of the same matter Lagrangian to $f_{\mu \nu}$ is not possible as it will generally reintroduce the BD ghost [65 67. Even though this will not happen if a different matter Lagrangian (an unobservable dark sector) is coupled to $f_{\mu \nu}$, we will usually meet a new fine tuning [68] that would make the theory less appealing. One way out would be a coupling through a new composite metric that is constructed such that it avoids the BD ghost [65]. This choice would lead to viable, self-accelerating backgrounds [69] but still does not yield a realistic cosmological evolution at the linear level [70, 71].

\section{CONCLUSIONS}

We have analyzed the behavior of tensor perturbations in the infinite-branch bigravity (IBB) model and the signatures they produce on the CMB. In this model the reference metric contracts at early times, causing tensor perturbations in this metric to grow rapidly. These modes have ample time to grow since the end of inflation. However, a consistent analysis of linear perturbations and CMB spectra cannot include the regime in which perturbations become non-linear. We then assume that this growth stops when perturbations become non-linear, with an amplitude saturated at a value of order unity. The coupling between the two metrics produces in turn a growth of the tensor perturbations in $g$. If the coupling is weak enough at early times, the growing mode will in principle propagate to the physical metric only after some time. Our first objective has been then to check whether this effect is late enough to keep the spectra of tensor perturbations compatible with present CMB data. Our conclusion is that, even when perturbations remain below order unity, they are still large enough to have a twofold impact on CMB spectra: first, the tensor modes provide a large contribution to the TT, EE and TE spectra which is orders of magnitude larger than the scalar contribution; second, the tensor modes induce a strong B-mode polarization on the CMB. Both effects dominate on the largest angular scales and are incompatible with observations from CMB experiments.

Varying the IBB parameter $\left(\beta_{1}\right.$, with $\beta_{4}$ being derived from it via Eq. (24) ) or other cosmological parameters offers little help in reconciling IBB bigravity theory with observations. We further explore five scenarios in which the theory might be rendered viable:

1. Lowering the energy scale of inflation. If the energy scale of inflation is very small, around $1 \mathrm{GeV}$, the tensor modes are naturally suppressed and the growing mode has less time to grow until recombination or reionization: 
the combined effect makes the IBB model acceptable without any change. If one invokes the freezing of the growing mode when it reaches non-linearity, then the inflationary energy scale can be increased up to $25 \mathrm{GeV}$ roughly. Such a scale is much lower than the one predicted in simple slow roll inflation but could in principle be achieved in alternative scenarios [58].

2. Fine-tuning the initial conditions. If $h_{f}^{\prime}$ is very small at early times, the perturbations will not have reached the non-linear value at late times, when the coupling to $g$ becomes important. This requires a fabulous degree of fine-tuning, of one part in $10^{26}$ at $z=10^{10}\left(10^{28}\right.$ when TT modes are considered). For this solution to work beyond the linear approximation, it is necessary that non-linear sources in the equation for $h_{f}$ are suppressed at early times. Even if stable agains non-linear corrections, fine tuning the initial conditions for $h_{f}$ seems a highly ad-hoc requirement for the theory in the absence of a mechanism, perhaps generalizing inflation, able to naturally produce such small initial conditions. As mentioned in the previous point, this fine-tuning occurs naturally only in the case of very low-energy inflation.

3. Lowering the non-linear cutoff. IBB becomes safe if $h_{f}$ stabilizes at a value smaller than unity due to non-linear effects. This requires the non-linear effects to act at most when $h_{f} \lesssim 10^{-3}$ (and $\lesssim 10^{-4}$ when TT modes are considered).

4. Adding a cosmological constant. In that case the theory still describes a massive graviton, although it would not be responsible for the acceleration of the universe. From a purely cosmological point of view, the model will be very similar to $\Lambda$ CDM.

5. Modifying the theory. One possibility is to allow for a time dependence to the theory parameters $\beta_{1}, \beta_{4}$ in the tensor perturbation equations. This phenomenological parametrization of modified IBB allows to satisfy CMB data if the parameters become non-zero only after $z=1000$.

While our assumption stops the growth of perturbations when they become non-linear, a full analysis would require an understanding of the actual non-linear behaviour of perturbations, which could be used to exclude or validate such scenarios in a fully consistent way.

Finding a modification of bigravity that overcomes the difficulties of the growing modes could be possible within the framework of generalized massive gravity [61. In this class of theories, the interaction terms are given an additional dependence in the Stückelberg fields, which allows the couplings to vary over time without introducing additional degrees of freedom. Another possibility in this direction is allowing a composite coupling that involves both tensor, possibly on an equal footing. Theories with additional degrees of freedom, such as scalar-bitensor or multigravity, might as well prove useful to solve the problem of growing modes. More exotic modifications of the theory remain to be explored.

Constructing a viable theory of massive gravity has proven to be a challenge. Only after eight decades could the linear Fierz-Pauli theory be generalized to a ghost-free non-linear completion, albeit one that forbids any interesting cosmological solution. This difficulty could be overcome by giving a kinetic term to the reference metric, allowing the existence of accelerating cosmologies at the price of two additional degrees of freedom, corresponding to a massless tensor. Of all the five-parameter set of bigravity theories, only the two-parameter IBB family is able to accelerate the universe with neither a cosmological constant nor scalar instabilities. Yet, such a theory is affected by growing modes that generically spoil the predictions of the cosmic microwave background. The results presented here represent a setback for the simple and appealing self-accelerating bigravity paradigm, a paradigm that, unless saved by non-linear effects or a tiny amplitude of the initial conditions, will have to be abandoned.

\section{Acknowledgments}

We thank Yashar Akrami, Ruth Durrer, Pedro Ferreira, Daniel G. Figueroa, Fawad Hassan, Macarena Lagos, Gerasimos Rigopoulos, Angnis Schmidt-May, Adam Solomon and Christof Wetterich for useful discussions. This research has been supported by DFG through the grant TRR33 "The Dark Universe". F.K. acknowledges support from the Graduate College "Astrophysics of fundamental probes of gravity".

[1] S. Perlmutter, G. Aldering, G. Goldhaber, R. A. Knop, P. Nugent, P. G. Castro, S. Deustua, S. Fabbro, A. Goobar, D. E. Groom, et al., The Astrophysical Journal 517, 21 (1998), 9812133. I] 
[2] A. G. Riess, A. V. Filippenko, P. Challis, A. Clocchiatti, A. Diercks, P. M. Garnavich, R. L. Gilliland, C. J. Hogan, S. Jha, R. P. Kirshner, et al., The Astronomical Journal 116, 1009 (1998), 9805201v1.

[3] L. Anderson, É. Aubourg, S. Bailey, F. Beutler, V. Bhardwaj, M. Blanton, A. S. Bolton, J. Brinkmann, J. R. Brownstein, A. Burden, et al., Mon. Not. Roy. Astron. Soc. 441, 24 (2014), 1312.4877.

[4] M. Betoule, R. Kessler, J. Guy, J. Mosher, D. Hardin, R. Biswas, P. Astier, P. El-Hage, M. Konig, S. Kuhlmann, et al., Astronomy \& Astrophysics 568, A22 (2014), 1401.4064.

[5] F. Beutler, S. Saito, H.-J. Seo, J. Brinkmann, K. S. Dawson, D. J. Eisenstein, A. Font-Ribera, S. Ho, C. K. McBride, F. Montesano, et al., Mon. Not. Roy. Astron. Soc. 443, 1065 (2014), 1312.4611.

[6] L. Samushia, B. A. Reid, M. White, W. J. Percival, A. J. Cuesta, G.-B. Zhao, A. J. Ross, M. Manera, É. Aubourg, F. Beutler, et al., Mon. Not. Roy. Astron. Soc. 439, 3504 (2014), 1312.4899.

[7] C. Heymans, L. Van Waerbeke, L. Miller, T. Erben, H. Hildebrandt, H. Hoekstra, T. D. Kitching, Y. Mellier, P. Simon, C. Bonnett, et al., Mon. Not. Roy. Astron. Soc. 427, 146 (2012), 1210.0032.

[8] Planck Collaboration, P. A. R. Ade, N. Aghanim, M. Arnaud, M. Ashdown, J. Aumont, C. Baccigalupi, A. J. Banday, R. B. Barreiro, N. Bartolo, et al., ArXiv e-prints (2015), 1502.01590. II

[9] Planck Collaboration, P. A. R. Ade, N. Aghanim, M. Arnaud, M. Ashdown, J. Aumont, C. Baccigalupi, A. J. Banday, R. B. Barreiro, J. G. Bartlett, et al., ArXiv e-prints (2015), 1502.01589. I]

[10] L. Amendola et al. (Euclid Theory Working Group) (2012), 1206.1225. II]

[11] L. Amendola and S. Tsujikawa, Dark Energy: Theory and Observations (Cambridge University Press, 2010), ISBN 0521516005. I]

[12] T. Clifton, P. G. Ferreira, A. Padilla, and C. Skordis, Phys.Rept. 513, 1 (2012), 1106.2476. I]

[13] G. W. Horndeski, Int.J.Th.Phys. 10, 363 (1974). I]

[14] C. Deffayet, X. Gao, D. Steer, and G. Zahariade, Phys.Rev. D84, 064039 (2011), 1103.3260. I]

[15] M. Zumalacarregui and J. Garcia-Bellido, Phys.Rev. D89, 064046 (2014), 1308.4685. I]

[16] J. Gleyzes, D. Langlois, F. Piazza, and F. Vernizzi (2014), 1404.6495. I]

[17] M. Fierz and W. Pauli, Proc.Roy.Soc.Lond. A173, 211 (1939). I]

[18] D. G. Boulware and S. Deser, Phys. Rev. D 6, 3368 (1972). I

[19] C. de Rham, G. Gabadadze, and A. J. Tolley, Physical Review Letters 106, 231101 (2011), 1011.1232. I]

[20] S. F. Hassan and R. A. Rosen, Physical Review Letters 108, 041101 (2012), 1106.3344. I]

[21] G. D'Amico, C. de Rham, S. Dubovsky, G. Gabadadze, D. Pirtskhalava, and A. J. Tolley, Phys. Rev. D 84, 124046 (2011), 1108.5231. I

[22] S. F. Hassan and R. A. Rosen, Journal of High Energy Physics 2, 126 (2012), 1109.3515. I] II]

[23] S. F. Hassan and R. A. Rosen, Journal of High Energy Physics 4, 123 (2012), 1111.2070.

[24] S. F. Hassan, R. A. Rosen, and A. Schmidt-May, Journal of High Energy Physics 2, 26 (2012), 1109.3230. I. II]

[25] K. Hinterbichler, Reviews of Modern Physics 84, 671 (2012), 1105.3735. I]

[26] C. de Rham, ArXiv e-prints (2014), 1401.4173. I

[27] M. von Strauss, A. Schmidt-May, J. Enander, E. Mörtsell, and S. Hassan, Journal of Cosmology and Astroparticle Physics 2012, 042 (2012). I II

[28] Y. Akrami, T. S. Koivisto, and M. Sandstad, Journal of High Energy Physics 3, 99 (2013), 1209.0457. II] II] II

[29] F. Koennig, A. Patil, and L. Amendola, Journal of Cosmology and Astroparticle Physics 2014, 029 (2014), 1312.3208. I] II] II] VC

[30] F. Könnig and L. Amendola (2014), 1402.1988. I] II]

[31] D. Comelli, M. Crisostomi, and L. Pilo, JHEP 1206, 085 (2012), 1202.1986. I] III] III]

[32] A. De Felice, A. E. Gumrukcuoglu, S. Mukohyama, N. Tanahashi, and T. Tanaka (2014), 1404.0008. I]

[33] M. Berg, I. Buchberger, J. Enander, E. Mortsell, and S. Sjors, JCAP 1212, 021 (2012), 1206.3496. I]

[34] A. R. Solomon, Y. Akrami, and T. S. Koivisto, ArXiv e-prints (2014), 1404.4061. I]

[35] F. Koennig, Y. Akrami, L. Amendola, M. Motta, and A. R. Solomon, Phys.Rev. D90, 124014 (2014), 1407.4331. I] II] II] II

[36] M. Lagos and P. G. Ferreira, JCAP 1412, 026 (2014), 1410.0207. I] III] III

[37] J. Enander, Y. Akrami, E. Mortsell, M. Renneby, and A. R. Solomon (2015), 1501.02140.

[38] J. Enander and E. Mortsell, JHEP 1310, 031 (2013), 1306.1086. I]

[39] G. Cusin, R. Durrer, P. Guarato, and M. Motta (2014), 1412.5979. I] III, III, III C, V A

[40] S. Dubovsky, R. Flauger, A. Starobinsky, and I. Tkachev, Phys.Rev. D81, 023523 (2010), 0907.1658. I

[41] M. Berg, I. Buchberger, J. Enander, E. Mörtsell, and S. Sjörs, JCAP 12, 021 (2012), 1206.3496. II]

[42] D. Comelli, M. Crisostomi, F. Nesti, and L. Pilo, Journal of High Energy Physics 3, 67 (2012), 1111.1983. II]

[43] T. L. Smith, M. Kamionkowski, and A. Cooray, Phys.Rev. D73, 023504 (2006), astro-ph/0506422. IIIB

[44] G. German, G. G. Ross, and S. Sarkar, Nucl.Phys. B608, 423 (2001), hep-ph/0103243. III C

[45] R. Allahverdi, K. Enqvist, J. Garcia-Bellido, and A. Mazumdar, Phys.Rev.Lett. 97, 191304 (2006), hep-ph/0605035.

[46] S. Choudhury, A. Mazumdar, and S. Pal, JCAP 1307, 041 (2013), 1305.6398. III C

[47] L. Amendola, M. Baldi, and C. Wetterich, Phys.Rev. D78, 023015 (2008), 0706.3064. IIID

[48] V. Pettorino, N. Wintergerst, L. Amendola, and C. Wetterich (2010), 1009.2461. IIID] IIID

[49] P. Ade et al. (Planck Collaboration) (2015), 1502.01590. IV]

[50] A. Lewis and S. Bridle, Phys. Rev. D66, 103511 (2002), astro-ph/0205436. IV]

[51] D. Blas, J. Lesgourgues, and T. Tram, JCAP 1107, 034 (2011), 1104.2933. IV

[52] P. Ade et al. (Planck Collaboration) (2015), 1502.01589. IV 
[53] R. Adam et al. (Planck Collaboration) (2015), 1502.01582. IV

[54] C. L. Bennett, D. Larson, J. L. Weiland, N. Jarosik, G. Hinshaw, N. Odegard, K. M. Smith, R. S. Hill, B. Gold, M. Halpern, et al., ArXiv e-prints (2012), 1212.5225. IV

[55] D. Barkats et al. (BICEP1 Collaboration) (2013), 1310.1422. IV]

[56] P. Ade et al. (BICEP2 Collaboration, Planck Collaboration), Phys.Rev.Lett. (2015), 1502.00612. IV]

[57] R. H. Becker et al. (SDSS Collaboration), Astron.J. 122, 2850 (2001), astro-ph/0108097. V]

[58] R. Kallosh, L. Kofman, and A. D. Linde, Phys.Rev. D64, 123523 (2001), hep-th/0104073. V A, 1

[59] A. Vainshtein, Phys.Lett. B39, 393 (1972). VB

[60] E. Babichev and C. Deffayet, Class.Quant.Grav. 30, 184001 (2013), 1304.7240. VB

[61] C. de Rham, M. Fasiello, and A. J. Tolley, Int.J.Mod.Phys. D23, 3006 (2014), 1410.0960. V C VI

[62] H. Nersisyan, Y. Akrami, and L. Amendola (2015), 1502.03988. V C

[63] Y. Akrami, T. S. Koivisto, D. F. Mota, and M. Sandstad, JCAP 10, 046 (2013), 1306.0004. V C

[64] Y. Akrami, T. S. Koivisto, and A. R. Solomon (2014), 1404.0006. V C

[65] C. de Rham, L. Heisenberg, and R. H. Ribeiro (2014), 1408.1678. V C, V C

[66] Y. Yamashita, A. De Felice, and T. Tanaka, ArXiv e-prints (2014), 1408.0487.

[67] J. Noller and S. Melville, ArXiv e-prints (2014), 1408.5131. V C

[68] K. Aoki and K.-i. Maeda, Phys.Rev. D90, 124089 (2014), 1409.0202. V C

[69] J. Enander, A. R. Solomon, Y. Akrami, and E. Mörtsell (2014), 1409.2860. V C

[70] A. E. Gumrukcuoglu, L. Heisenberg, S. Mukohyama, and N. Tanahashi (2015), 1501.02790. V C

[71] D. Comelli, M. Crisostomi, K. Koyama, L. Pilo, and G. Tasinato (2015), 1501.00864. V C 